\title{
Digital Certificate System for Verification of Educational Certificates using Blockchain
}

\author{
${ }^{1}$ Vipul Badhe, ${ }^{2}$ Pooja Nhavale, ${ }^{3}$ Sonal Todkar, ${ }^{4}$ Prajakta Shinde, ${ }^{5}$ Prof. Kiran Kolhar \\ 1,2,3,4 Students and ${ }^{5}$ Assistant Professor
}

Department of Computer Engineering, Dr. D Y Patil School of Engineering \& Technology, Savitribai Phule Pune University, Pune, Maharashtra, India

\begin{abstract}
Article Info
Volume 7, Issue 5

Page Number: 45-50

Publication Issue :
\end{abstract}

September-October-2020

\section{Article History}

Accepted : 05 Sep 2020

Published : 15 Sep 2020

\section{ABSTRACT}

While the number of universities, tertiary education students and number of graduates per year constantly increase, the need to easily verify degree certificates generates new business opportunities. In this paper we project two financial models balancing where the price for the service is balanced between the graduate and the employer as the main stakeholders of that service. Students demand a proof-of-certification at low cost and easy to check, employers also demand quick and trustable verification of degrees when recruiting. As large number of students graduate every year, the problem of fake certificates is a big issue. One can easily get fake certificates in India. Companies hiring thousands of fresher spend large amount of money to get the educational certificates and transcripts verified of applicants. A Digital Certificate using blockchain technology can address this problem. Blockchain is a decentralized distributed digital ledger collectively maintained by a network of computers, called nodes. The data in the blockchain cannot be modified by a person without the consent of everyone else who maintains the records. This makes the data secure.

Keywords: Blockchain, Document Verification, Digital Certificate, distributed, Preprocessing

\section{INTRODUCTION}

The blockchain technology opens today opportunities to deliver new business models on quite consolidated markets. The use of blockchain in the education sector is one of the most challenging areas where results in the mid and long term can be achieved. The easy, trustable and cheap verification of official documents, such as university degrees, is one of the areas where blockchain can provide a timely and solid solution thanks to the use of widely extended that offer a stable public blockchain that can be used for secondary uses such as a verification tool in several markets. Here, the selection of an appropriate public blockchain in terms of availability, flexibility and cost 
is crucial to develop a sustainable business model on top.

As the data used for scientific research increases exponentially, ensuring information quality and preventing data manipulation has emerged as an important factor in validating the research results. Graduation certificates and transcripts contain information confidential to the individuals and should not be easily accessible to others. Hence, there is a high need for a mechanism that can guarantee that the information in such a document is original, which means that document has originated from an authorized source and is not fake. In addition, the information in the document should be confidential so that it can only be viewed by authorized persons. Blockchain technology is used to reduce the incidence of certificate forgeries and ensure that the security, validity and confidentiality of graduation certificates would be improved. Technologies exist in related domains, such as digital signatures, which are used in e-documents to provide authentication, integrity, and nonrepudiation. However, for the requirements of an e-qualification certificate, it has critical security holes and missing functions: for example, it uses the keys to verify the modification of the document, but doesn't start the validation of the public key certificates' status automatically. This may result in a forgery being accepted if the key has been compromised. Furthermore, even the signer's public key certificate has been validated, but the signed document itself hasn't. In our case of an equalification certificate, the signed document itself is also a certificate, which may have a valid period (e.g. The problem we are dealing with is a (certificate) issue, therefore, a simple digital signing of the document alone doesn't solve the problem.

\section{PROBLEM STATEMENT}

In Existing system, the problem of fake certificates is a big issue. Companies hiring thousands of fresher spend large amount of money to get the educational certificates and transcripts verified of applicants. To address this problem, we have proposed an idea of Digital Certificate System for verification of educational certificates using blockchain technology.

\section{LITERATURE SURVEY}

The Jiin-Chiou Chen, Narn-Yih Lee, Chien Chi, and Yi-Hua Chen "Blockchain and Smart Contract for Digital Certificate" [1] In order to solve the problem of certificate frogery, the digital certificate system based on blockchain technology would be proposed. Due to the unmodifiable property of blockchain, the digital certificate with anti-counterfeit and verifiability could be made. The procedure given for issuing the digital certificate in this system is as follows. Firstly, the generation of an electronic file of a paper certificate accompanying other related data will be done into the database, also calculation of the electronic file for its hash value will be done. Finally, the hash value will be stored into the block in the chain system. A QR-code and inquiry string code related to the certificate will be generated by the system to affix to the paper certificate. A demand unit will be provided to verify the authenticity of the paper certificate by scanning through mobile phones or by website inquiries. Due to the unmodifiable properties of the blockchain, the system enhances the credibility of various paper-based certificates and also electronically minimizes the loss risks of various types of certificates.

Austin Draper, Aryan Familrouhani, Devin Cao, Tevisophea Heng, Wenlin Han "Security Applications and Challenges in Blockchain" [2] Blockchain technology is very much popular but still a highly misunderstood concept that is used today and will be used in the future applications. To improve the security and privacy, many applications adopt Blockchain. However, there are intrinsic drawbacks and emerging challenges. In this paper, we study 
popular security applications in Blockchain, their major problems, as well as other challenges in Blockchain which allows future research to be conducted more efficiently.

Marco Baldi, Franco Chiaraluce, Emanuele Frontoni, Giuseppe Gottardi, Daniele Sciarroni and Luca Spalazzi Certificate "Validation through Public Ledgers and Blockchain" [3] Public key infrastructures (PKIs) are of crucial importance for the life of online services relying on certificate-based authentication, like e-commerce, e-government, online banking, as well as e-mail, social networking, cloud services and many others. One of the main points of failure of modern PKIs concerns reliability and security of certificate revocation lists, that must be available and authentic any time a certificate is used. Classically, the CRL for a set of certificates is maintained by the same (and sole) certification authority (CA) that issued the certificates, and this introduces a single POF in the system. We address this issue by proposing a solution in which multiple CAs share a public, decentralized and robust ledger where CRLs are collected. For this purpose, we consider the model of public ledgers based on blockchain, introduced for the use in cryptocurrencies, that is becoming a widespread solution for many online applications with stringent security and reliability requirements.

Santosh Pandey, Gopal ojha, Rohit Kumar and Bikesh Shresha "BlockSIM: A practical simulation tool for optimal network design, stability and planning" [4] In this paper they have introduced a BlockSIM, which is a comprehensive and open source blockchain system simulation tool. It can assist blockchain architects for better evaluation of the performance of planned private blockchain networks by running scenarios and deciding the optimal system parameters suited for their purposes. They have compared the results of their simulation with real blockchain networks and demonstrated that BlockSIM can be used effectively by architects of blockchain systems to plan and implement scalable, stable and resilient blockchain networks. Also a demonstration via a real life example is provided stating how the architects can apply BlockSIM to plan and design real-world blockchain systems.

Christopher Ehmke, Florian Wessling and Christoph M. Friedrich "Proof-of-Property - A Lightweight and Scalable Blockchain Protocol" [5] The approach proposed in this paper is based on the idea of Ethereum to keep the state of the system explicitly in the current block but further pursues this by including the relevant part of the current system state in new transactions as well. This enables other participants to validate incoming transactions without having to download the whole blockchain initially. Following this idea use cases can be supported that require scalable blockchain technology but not necessarily an indefinite and complete transaction history.

\section{S. Sunitha kumara, D. Saveetha "Blockchain and Smart} Contract for Digital Document Verification" [6] In the proposed system along with the degree certificate entire personality and behavior activities of the person using personal id will be uploaded in blockchain. Because of unmodifiable property it is stored in block chain. Initially the student will request for the ecertificate by uploading certificate or personal id to electronic certificate system. After requesting for ecertificate, the system will then review certificate from the university or schools or from organization and get the assurance and store the serial number and e-certificate to the block chain. The system will generate the $\mathrm{QR}$ code and send it to the user. When applying for company user will send only the certificate serial number and $Q R$ code received from the e-certificate system.

Arvind Ramachandran, Dr. Murat Kantarcioglu "Using Blockchain and smart contracts for secure data 
provenance management" [7] In this work, they leverage blockchain as a platform to provide trustworthy data provenance collection, verification and management. The developed system utilizes smart contracts and open provenance model (OPM) to record immutable data trails. The paper shows that proposed framework can efficiently and securely capture and validate provenance data, and prevent any malicious changes to the captured data as long as majority of the participants are honest.

Ahmed Ben Ayed "Secure storage service of electronic ballot system based on block chain algorithm" [8] In this paper, authors have leveraged the open source Blockchain technology to propose a design for a new electronic voting system that could be used in local or national elections. The Blockchain-based system will be secure, reliable, and anonymous and will help increase the number of voters as well as the trust of people in their governments.

\section{Kaidong Wu "An Empirical Study of Blockchain-based} Decentralized Applications" [9] This paper presents a comprehensive empirical study on an extensive dataset of 734 dapps that are collected from three popular open dapp marketplaces, i.e., ethereum, state of the dapp, and DAppRadar. We analyze the popularity of dapps, and summarize the patterns of how smart contracts are organized in a dapp. Based on the findings, we draw some implications to help dapp developers and users better understand and deploy dapps.

Jialiang Chang, Bo Gao, Hao Xiao, Jun Sun and Zijiang Yang "sCompile: Critical Path Identification and Analysis for Smart Contracts" [10] In this work, an alternative approach to automatically identify critical program paths (with multiple function calls including inter-contract function calls) in a smart contract, rank the paths according to their criticalness, discard them if they are infeasible or otherwise present them with user friendly warnings for user inspection has been proposed. Identification of paths which involve monetary transaction as critical paths and prioritizing those which potentially violate important properties has been done. For scalability, symbolic execution techniques are only applied to top ranked critical paths. This approach has been implemented in a tool called sCompile, which has been applied to 36,099 smart contracts. The experiment results show that sCompile is efficient, i.e., 5 seconds on average for one smart contract

\section{PROPOSED WORK}

Nowadays the students achieve various educational certificates. Student produces these certificates while applying for jobs at public or private sectors, where all these certificates are needed to be verified manually. There can be incidents where students may produce the fake certificate and it is difficult to identify them. This problem of fake academic certificates has been a longstanding issue in the academic community. Because it is possible to create such certificates at low cost and the process to verify them is very complex, as they are manually needed to be verified. This problem can be solved by storing the digital certificates on the Blockchain.

To create the blockchain based unmodifiable certificates, initially the university needs to get registered. Any transaction can be sent through the wallet address of the registered university. Only the owner of the smart contract has the authority to add the universities. Once added the university, will be able to access the system and can create certificates with data fields. Each created certificate will be stored in the Inter planetary file system (IPFS). It will then return the unique hash generated using SHA-256 algorithm. This will serve as unique identity for each document. This generated hash and detail of certificates will be stored in the blockchain and the 
student will be provided with the resultant transaction id. Anyone can use this transaction id to verify the certificate details and can view the original copy of certificate using IPFS hash stored along with data. And it is not possible to modify this certificate or to create fake certificate using the same data. Hence with this we can solve the problem of certificate forgery.

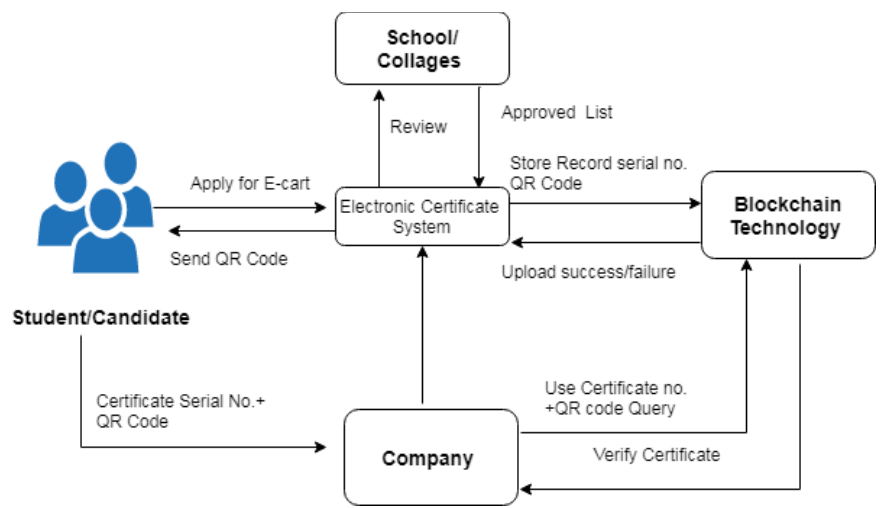

Fig.1: System Architecture

\section{CHALLENGES}

Data immutability is one of the main features of blockchain. It serves as a large public ledger where node in network verifies and save the same data. The process of certificate generation is open and transparent system where any organization can verify information of any certificate using this system. In challenges the system helps in eradicating problems of fake certificates.

\section{CONCLUSION}

Various technologies have been discussed to reduce the incidence of certificate forgeries and ensure that the security, validity and confidentiality of graduation certificates, even though there are many limitations regarding the security and privacy of data. A new blockchain-based system reduces the certificate forgery. Automated certificate granting is open and transparent in the system. Companies or organizations can thus inquire for information on any certificate from the system. The proposed system, cuts down the management cost, prevents document forgery and provides accurate and reliable information on digital certificates.

\section{ACKNOWLEDGMENT}

We genuinely thank all the Staff of Dr. D Y Patil School of Engineering and Technology, Lohegaon, Pune for their kind help and co-operation throughout our study period.

Also we are extremely thankful to the researchers and the publishers for making their resources available.

\section{REFERENCES}

[1]. Jiin-Chiou Chen, Narn-Yih Lee, Chien Chi, and Yi-Hua Chen "Blockchain and Smart Contract for Digital Certificate" Proceedings of IEEE International Conference on Applied System Innovation 2018 IEEE ICASI 2018- Meen, Prior \& Lam (Eds)

[2]. Austin Draper, Aryan Familrouhani, Devin Cao, Tevisophea Heng, Wenlin Han "Security Applications and Challenges in Blockchain" Published in IEEE International Conference on Consumer Electronics (ICCE) 2019

[3]. Marco Baldi, Franco Chiaraluce, Emanuele Frontoni, Giuseppe Gottardi, Daniele Sciarroni and Luca Spalazzi Certificate "Validation through Public Ledgers and Blockchains" In Proceedings of the First Italian Conference on Cybersecurity (ITASEC17) 2017

[4]. Neethu Gopal, Vani V Prakash "Survey on Blockchain Based Digital Certificate System" International Research Journal of Engineering and Technology (IRJET) Nov 2018 
[5]. Santosh Pandey, Gopal ojha, Rohit Kumar and Bikesh Shresha "BlockSIM: A practical simulation tool for optimal network design, stability and planning" 2019 IEEE International Conference on Blockchain and Cryptocurrency (ICBC).

[6]. Christopher Ehmke, Florian Wessling and Christoph M. Friedrich "Proof-of-Property - A Lightweight and Scalable Blockchain Protocol" 2018 IEEE/ACM 1st International Workshop on Emerging Trends in Software Engineering for Blockchain (WETSEB).

\section{Cite this article as :}

Vipul Badhe, Pooja Nhavale, Sonal Todkar, Prajakta Shinde, Prof. Kiran Kolhar, "Digital Certificate System for Verification of Educational Certificates using Blockchain ", International Journal of Scientific Research in Science and Technology (IJSRST), Online ISSN : 2395-602X, Print ISSN : 2395-6011, Volume 7 Issue 5, pp. 45-50, September-October 2020. Available at doi : https://doi.org/10.32628/IJSRST20758 Journal URL : http://ijsrst.com/IJSRST20758 